\title{
Managing acute myocardial infarction in patients with COVID-19 at a cardiac catheterisation laboratory
}

Alan KC Chan ${ }^{1 *}$, MB, BS, FHKAM (Medicine), CF Tsang ${ }^{1}$, MB, ChB, FHKAM (Medicine), SF Chui ${ }^{1}$, MB, ChB, FHKAM (Medicine), Eric CY Wong ${ }^{1}$, MB, BS, FHKAM (Medicine), SY Au², MB, BS, FHKAM (Medicine), George WY Ng², MB, BS, FHKAM (Medicine), KT Chan ${ }^{1}$, MB, BS, FRCP, Michael KY Lee ${ }^{1}$, MB, BS, FRCP

${ }^{1}$ Department of Medicine, Queen Elizabeth Hospital, Hong Kong

${ }^{2}$ Intensive Care Unit, Queen Elizabeth Hospital, Hong Kong

*Corresponding author: chkmkckk@gmail.com

\section{Coronavirus disease 2019 and cardiovascular disease}

Patients with cardiovascular disease who develop coronavirus disease 2019 (COVID-19) have a higher risk of mortality. They can develop various cardiovascular complications during their course of disease including acute myocardial infarction, myocarditis mimicking ST elevation myocardial infarction (STEMI), stress cardiomyopathy, coronary spasm, or myocardial injury. ${ }^{1}$ Patients presenting with STEMI pose a substantial dilemma to healthcare providers in the COVID-19 era. Patients with STEMI typically require emergency reperfusion therapy which include primary percutaneous coronary intervention (PPCI) in the cardiac catheterisation laboratory (CCL) or thrombolytic therapy. The former approach is the preferred strategy according to latest clinical evidence., ${ }^{2,3}$ After STEMI is diagnosed, PPCI must be performed quickly, and COVID-19 status might not be available. Some patients who present with cardiogenic shock shortly after STEMI or failed thrombolysis may also require emergency revascularisation in CCL. Most CCL facilities in Hong Kong are not equipped with negative pressure ventilation systems and may share common ventilation with the control room next door where healthcare providers are working. Various international cardiology professional societies have issued recommendations on treating this group of patients. The Chinese Society of Cardiology, for example, recommends a conservative approach including thrombolytic therapy and guidelinedirected medical therapies for patients with STEMI unless clinically unstable ${ }^{4}$; in contrast, the American College of Cardiology recommends the PPCI approach where possible. ${ }^{5}$

There have been advances in treating patients with cardiogenic shock using invasive mechanical circulatory support devices. ${ }^{6}$ Furthermore, during the severe acute respiratory syndrome outbreak in 2003, doctors in Hong Kong described their approach to treating patients with the CCL using full personal protective equipment and portable machines to simulate a negative pressure environment. ${ }^{7}$ More recently, the American College of Cardiology's Interventional Council and Society for Cardiovascular Angiography and Interventions have described CCL preparation during the COVID-19 pandemic. ${ }^{8}$

\section{Change in practice}

In our unit, PPCI is offered to all patients with STEMI unless contra-indicated. It remains the standard of practice in our referring hospital for eligible patients with STEMI during COVID-19 period unless they have signs or symptoms suggestive of COVID-19, including fever, abnormal lung infiltrates, or contact history. For patients with suspected COVID-19, thrombolytic therapy is given, and earlier PPCI arranged only after COVID-19 has been ruled out.

Good preparation of the medical team and the patients is the key to success in providing timely invasive cardiac interventions, while minimising the chance of in-hospital transmission of the virus. Patients presenting with STEMI are usually in an unstable condition with high possibility of deterioration resulting from heart failure or malignant arrhythmia. These patients may also develop desaturation requiring intubation or ventricular arrhythmia requiring defibrillation in the CCL during PPCI. Patients with COVID-19 with borderline respiratory function receive early intubation in the isolation area first; and invasive mechanical circulatory support devices are initiated early for patients with high risk of haemodynamic collapse that may otherwise require cardiopulmonary resuscitation in the CCL. The CCL environment has been checked by the hospital engineering team, who confirmed that the CCL procedural room does not share the same ventilation 
system with the control room or other CCL areas that would otherwise contaminate the environment and risk infecting unprotected medical staff. In one designated CCL procedural room in our hospital, two high-efficiency particulate air filters were installed and the ventilation system was upgraded in order to provide temporary negative pressure environment when needed and to achieve more than 12 air changes per hour. This designated CCL procedural room was left with essential equipment only to avoid contamination of other consumable items. Fixed equipment, such as the X-ray machine and cabinets were adequately covered before procedures. As training and preparation, the cardiac and anaesthetic teams simulated the PPCI of an unstable patient with COVID-19 in the CCL who required intubation and cardiopulmonary resuscitation. In order to limit the number of personnel involved, two interventional cardiologists and one CCL nurse perform the PPCI procedure. All personnel in the CCL wear lead aprons and full personal protective equipment-N95 respirator, cap, face shield, gown, surgical gloves, and boots-in accordance with local infection control guidelines. Immediate showering after procedures in a sanitary area of the CCL was provided. The designated CCL room had terminal cleaning after the procedure. The logistics of transferring the critically ill patient with COVID-19 was discussed and organised with staff from the intensive care unit in advance, to minimise emergent aerosol-generating procedures in the CCL.

\section{Experience and conclusions}

We have performed three coronary angiograms, two percutaneous coronary interventions, and one endomyocardial biopsy for three patients with confirmed COVID-19. All three patients were in critical condition and required mechanical ventilatory support. One patient required venoarterial extracorporeal membrane oxygenation for circulatory support for fulminant cardiogenic shock.

Our approach may provide useful information to other hospitals providing emergency interventional cardiology service for patients with suspected or confirmed COVID-19. Teamwork and close communication between cardiologists and intensive care unit specialists are essential before performing invasive cardiac procedures in the CCL.

\section{Author contributions}

All authors contributed to the concept or design of the study, acquisition of the data, analysis or interpretation of the data, drafting of the manuscript, and critical revision of the manuscript for important intellectual content. All authors had full access to the data, contributed to the study, approved the final version for publication, and take responsibility for its accuracy and integrity.

\section{Conflicts of interest}

The authors have no conflicts of interest to disclose.

\section{Funding/support}

This commentary received no specific grant from any funding agency in the public, commercial, or not-for-profit sectors.

\section{References}

1. Nishiga M, Wang DW, Han Y, Lewis DB, Wu JC. COVID-19 and cardiovascular disease: from basic mechanisms to clinical perspectives. Nat Rev Cardiol 2020;17:543-58.

2. Ibanez B, James S, Agewall S, et al. 2017 ESC Guidelines for the management of acute myocardial infarction in patients presenting with ST-segment elevation: The Task Force for the management of acute myocardial infarction in patients presenting with ST-segment elevation of the European Society of Cardiology (ESC). Eur Heart J 2018;39:119-77.

3. Levine GN, Bates ER, Blankenship JC, et al. 2015 ACC/ AHA/SCAI focused update on primary percutaneous coronary intervention for patients with ST-elevation myocardial infarction: an update of the 2011 ACCF/AHA/ SCAI guideline for percutaneous intervention and the 2013 ACCF/AHA guideline for the management of ST-elevation myocardial infarction. J Am Coll Cardiol 2016;67:1235-50.

4. Han $\mathrm{Y}$, Zeng $\mathrm{H}$, Jiang $\mathrm{H}$, et al. CSC expert consensus on principles of clinical management of patients with severe emergent cardiovascular diseases during the COVID-19 epidemic. Circulation 2020;141:e810-6.

5. Mahmud E, Dauerman HL, Welt FG, et al. Management of acute myocardial infarction during the COVID-19 pandemic a position statement from the Society for Cardiovascular Angiography and Interventions (SCAI), the American College of Cardiology (ACC), and the American College of Emergency Physicians (ACEP). J Am Coll Cardiol 2020;76:1375-84.

6. Vahdatpour C, Collins D, Goldberg S. Cardiogenic shock. J Am Heat Assoc 2019;8:e011991.

7. Tsui KL, Li SK, Li MC, et al. Preparedness of the cardiac catheterization laboratory for severe acute respiratory syndrome (SARS) and other epidemics. J Invasive Cardiol 2005;17:149-52.

8. Welt FG, Shah PB, Aronow HD, et al. Catheterization laboratory considerations during the coronavirus (COVID-19) pandemic. From the ACC's interventional council and SCAI. J Am Coll Cardiol 2020;75:2372-5. 